\title{
Article
}

\section{Familial Multiple Coagulation Factor Deficiencies (FMCFDs) in a Large Cohort of Patients-A Single-Center Experience in Genetic Diagnosis}

\author{
Barbara Preisler ${ }^{1,+}{ }^{+}$Behnaz Pezeshkpoor ${ }^{1,+}+\mathbb{D}$, Atanas Banchev ${ }^{2}\left(\mathbb{D}\right.$, Ronald Fischer ${ }^{3}$, Barbara Zieger ${ }^{4}$, Ute Scholz $^{5}$, \\ Heiko Rühl ${ }^{1}$, Bettina Kemkes-Matthes ${ }^{6}$, Ursula Schmitt ${ }^{7}$, Antje Redlich ${ }^{8}{ }^{\circledR}$, Sule Unal ${ }^{9}{ }^{\circledR}$, Hans-Jürgen Laws ${ }^{10}$, \\ Martin Olivieri ${ }^{11}{ }^{\mathbb{B}}$, Johannes Oldenburg ${ }^{1}(\mathbb{1})$ and Anna Pavlova ${ }^{1, *}$
}

check for

updates

Citation: Preisler, B.; Pezeshkpoor, B.; Banchev, A.; Fischer, R.; Zieger, B.; Scholz, U.; Rühl, H.; Kemkes-Matthes, B.; Schmitt, U.; Redlich, A.; et al. Familial Multiple Coagulation Factor Deficiencies (FMCFDs) in a Large Cohort of Patients-A Single-Center Experience in Genetic Diagnosis. J. Clin. Med. 2021, 10, 347. https:// doi.org/10.3390/jcm10020347

Received: 2 December 2020

Accepted: 14 January 2021

Published: 18 January 2021

Publisher's Note: MDPI stays neutral with regard to jurisdictional claims in published maps and institutional affiliations.

Copyright: (c) 2021 by the authors. Licensee MDPI, Basel, Switzerland. This article is an open access article distributed under the terms and conditions of the Creative Commons Attribution (CC BY) license (https:/ / creativecommons.org/licenses/by/ $4.0 /)$.
1 Institute of Experimental Hematology and Transfusion Medicine, University Clinic Bonn, 53127 Bonn, Germany; Barbara.Preisler@ukbonn.de (B.P.); Behnaz.Pezeshkpoor@ukbonn.de (B.P.); Heiko.Ruehl@ukbonn.de (H.R.); Johannes.Oldenburg@ukbonn.de (J.O.)

2 Department of Paediatric Haematology and Oncology, University Hospital "Tzaritza Giovanna-ISUL", 1527 Sofia, Bulgaria; bantschev@gmail.com

3 Hemophilia Care Center, SRH Kurpfalzkrankenhaus Heidelberg, 69123 Heidelberg, Germany; ronald.fischer@srh.de

4 Department of Pediatrics and Adolescent Medicine, University Medical Center-University of Freiburg, 79106 Freiburg, Germany; barbara.zieger@uniklinik-freiburg.de

5 Center of Hemostasis, MVZ Labor Leipzig, 04289 Leipzig, Germany; u.scholz@labor-leipzig.de

6 Hemostasis Center, Justus Liebig University Giessen, 35392 Giessen, Germany; bettina.kemkes-matthes@innere.med.uni-giessen.de

7 Center of Hemostasis Berlin, 10789 Berlin-Schöneberg, Germany; schmitt@coagumed.de

8 Pediatric Oncology Department, Otto von Guericke University Children's Hospital Magdeburg, 39120 Magdeburg, Germany; antje.redlich@med.ovgu.de

9 Division of Pediatric Hematology Ankara, Hacettepe University, 06100 Ankara, Turkey; suleunal@hacettepe.edu.tr

10 Department of Pediatric Oncology, Hematology and Clinical Immunology, University of Duesseldorf, 40225 Duesseldorf, Germany; laws@med.uni-duesseldorf.de

11 Pediatric Hemostasis and Thrombosis Unit, Department of Pediatrics, Pediatric Hemophilia Centre, Dr. von Hauner Children's Hospital, University Hospital, LMU Munich, 80337 Munich, Germany; martin.olivieri@med.uni-muenchen.de

* Correspondence: Anna.Pavlova@ukbonn.de; Tel.: +49-228-287-19711

+ These authors contributed equally to this work.

\begin{abstract}
Background: Familial multiple coagulation factor deficiencies (FMCFDs) are a group of inherited hemostatic disorders with the simultaneous reduction of plasma activity of at least two coagulation factors. As consequence, the type and severity of symptoms and the management of bleeding/thrombotic episodes vary among patients. The aim of this study was to identify the underlying genetic defect in patients with FMCFDs. Methods: Activity levels were collected from the largest cohort of laboratory-diagnosed FMCFD patients described so far. Genetic analysis was performed using next-generation sequencing. Results: In total, 52 FMCFDs resulted from coincidental co-inheritance of single-factor deficiencies. All coagulation factors (except factor XII (FXII)) were involved in different combinations. Factor VII (FVII) deficiency showed the highest prevalence. The second group summarized 21 patients with FMCFDs due to a single-gene defect resulting in combined FV/FVIII deficiency or vitamin K-dependent coagulation factor deficiency. In the third group, nine patients with a combined deficiency of FVII and FX caused by the partial deletion of chromosome 13 were identified. The majority of patients exhibited bleeding symptoms while thrombotic events were uncommon. Conclusions: FMCFDs are heritable abnormalities of hemostasis with a very low population frequency rendering them orphan diseases. A combination of comprehensive screening of residual activities and molecular genetic analysis could avoid underand misdiagnosis.
\end{abstract}

Keywords: blood coagulation disorders; combined deficiency of coagulation factors; genetic testing; NGS; thrombosis 


\section{Introduction}

Familial multiple coagulation factor deficiency (FMCFD) is a rare hemostatic disorder, characterized by the simultaneous reduction of plasma activity of more than one coagulation factor. FMCFDs are caused by genetic defects in one or more underlying genes. Earlier reports of deficiencies in various combinations of coagulation factors led to the classification of FMCFDs into three groups [1-3]. In the first group, FMCFDs arise from the coincidental inheritance of single-coagulation-factor deficiencies. This concomitant inheritance could involve two or more single hemorrhagic or thrombotic deficiencies alone or can represent a combination of hemorrhagic and thrombotic disorders [4-6]. The second group summarizes cases, where FMCFDs are caused by a single-gene defect. The best known, comprising the vast majority of reported cases of FMCFDs, are combined deficiency of factor V (FV) and factor VIII (FVIII) [7,8] and combined deficiency of vitamin $\mathrm{K}$-dependent clotting factors (VKCFD) $[9,10]$. The third group includes FMCFDs resulting from cytogenetic abnormalities [11].

Inherited deficiencies of single coagulation factors other than hemophilia A and B and von Willebrand disease are rare disorders, transmitted in an autosomal-recessive pattern. The deficiencies of fibrinogen, prothrombin, FV, factor VII (FVII), factor X (FX), factor XI (FXI), and factor XIII (FXIII) display a variable prevalence ranging from 1:500,000 to $1: 2,000,000[12,13]$. The clinical presentation of these disorders varies from asymptomatic, observed for heterozygotes, to severe phenotypes typical of homozygotes or compound heterozygotes [14]. Considering the rare prevalence of single-coagulation-factor deficiencies, combined deficiencies are much rarer except in some ethnic populations due to consanguineous marriages or founder variants $[6,15]$. Thus, FMCFDs present significant challenges in diagnosis and management and in many cases remain underdiagnosed.

Molecular diagnosis of the single-coagulation-factor deficiencies is based on the identification of pathogenic and likely pathogenic genetic variants in genes encoding corresponding coagulation factors [16]. Causative gene defects can be classified into genetic variants affecting protein biosynthesis/secretion, resulting in low antigen or activity in plasma, or genetic alterations leading to the secretion of a dysfunctional protein. In FMCFDs arising from the coincidental concomitant inheritance of separate coagulation-factor deficiencies, a combination of different disease-causing genetic changes in affected factors can be detected [17-19]. In FMCFDs arising from a single-gene defect, such as combined FV and FVIII deficiency and combined deficiency of the vitamin K-dependent factors, genetic variants in the genes encoding proteins responsible for the intracellular trafficking of FV and FVIII, (multiple coagulation factor deficiency 2 (MCFD2) and lectin mannose-binding 1 (LMAN1)) and genetic variants in genes encoding enzymes involved in post-translational modification and vitamin $\mathrm{K}$ metabolism ( $\gamma$-glutamylcarboxylase (GGCX) and vitamin $\mathrm{K}$ epoxide reductase complex subunit 1 (VKORC1)) are reported [7,20-22]. FMCFDs resulting from cytogenetic abnormalities due to the deletion of the end of chromosome 13 cause combined FVII and FX deficiency [18,23]. Similar findings have been reported with case(s) with combined hemophilia A and B [24].

While the diagnosis for a single-coagulation-factor deficiency is often straightforward, diagnosis of FMCFDs can be challenging due to high disease heterogeneity and often indistinct clinical and laboratory features. Frequently, the diagnosis can only be definite when genetic analysis is performed and defects in gene(s) leading to FMCFDs are identified. This paper summarizes genetic and laboratory data of the largest cohort of patients with FMCFDs.

\section{Experimental Section}

2.1. Patient Cohort

The study group was composed of 82 unrelated index patients (IPs) from centers in Germany, Switzerland, Turkey, and Lithuania. The inclusion criterion for all participants 
was the simultaneous reduction of plasma activity of at least two coagulation factors. The mean patients' age was 26 years, ranged from 3 months to 83 years. Forty-six of them $(56 \%)$ were male and $36(44 \%)$ were female. Patients' data were collected by contacting the primary care physicians via questionnaires. Residual coagulation factor activities were analyzed in local labs. The study was approved by the medical ethics committee of the Bonn University of Medical Sciences, and the patients and legal guardians signed a written consent according to the Declaration of Helsinki.

\subsection{Molecular Genetics Analyses}

All genetic analyses were performed in the Department of Molecular Hemostaseology, University Hospital Bonn. Genomic DNA was isolated from peripheral whole blood using the Blood Core Kit (Qiagen, Hilden, Germany). Molecular genetic analyses included the sequencing of all coding regions and intron/exon boundaries of the following genes: $F 2, F 5, F 7, F 8, F 9, F 10, F 11, F 13 A 1, F 13 B, F G A$ (fibrinogen alpha chain), FGB (fibrinogen beta chain), FGG (fibrinogen gamma chain), VWF, GGCX, VKORC1, LMAN1, MCFD2, SERPINC1 (serpin family C member 1, antithrombin), PROS1, and PROC.

The sequencing analyses were carried out on an ABI Prism 3130 genetic analyzer, for Sanger sequencing (Thermo Fisher Scientific, Langenselbold, Germany) and a MiniSeq genome sequencer (Illumina, Santa Clara, CA, USA) was used for next-generation sequencing (NGS). Data were evaluated by SeqScape Version 2.7 (Thermo Fisher Scientific) and SeqPilot (JSI medical systems, Ettenheim, Germany) software. Primers and conditions are available on request. For the description of sequence variations at the DNA and protein level, the guidelines of the Human Genome Variation Society (HGVS) were used. The genetic variant interpretation and criteria used to establish variant pathogenicity were performed according to the ACMG (American College of Medical Genetics) and AMP (Association for Molecular Pathology) guidelines for the interpretation of sequence variants [25]. The disease causality of genetic variants was compared in the Human Gene Mutation Database (HGMD) [26] and ClinGen database [27].

Large deletions and duplications were analyzed with multiplex ligation-dependent probe amplification (MLPA) analysis or copy number variation (CNV) analysis. MLPA was performed according to the manufacturer's recommendations, using SALSA MLPA Kits (MRC-Holland, Amsterdam, Netherlands). Amplification products were run on an ABI PRISM 3130XL DNA Sequencer (Thermo Fisher Scientific) with the GeneScan 500 ROX size standard (Thermo Fisher Scientific). Dosage analyses were performed by Coffalyser (V5.2) software (MRC-Holland). CNV evaluation was achieved by SeqPilot (JSI medical systems $\mathrm{GmbH})$.

\section{Results}

\subsection{Different Mechanisms Causing FMCFDs}

We investigated a total of 82 FMCFD index patients with almost exclusively laboratorydiagnosed simultaneous plasma activity reduction of at least two coagulation factors. According to the underlying genetic defects and current classification, patients were divided into three main groups.

3.1.1. Group I: Combined Deficiency Caused by Co-Inherited Deficiency of at Least Two Single Clotting Factors

In this group, we report 52 patients with co-inherited genetic defects in either two pro-coagulant or in two anti-coagulant genes. Patients bearing one genetic alteration in a pro-coagulant gene co-inherited with one genetic defect in an anti-coagulant gene were also included in this group.

In total, 44 patients were identified with a co-inheritance of two pro-coagulant factor deficiencies (Table 1a). Among all patients, the most common detected combined deficiency was that involving FVII. Decreased residual FVII plasma levels (mean $42 \mathrm{IU} / \mathrm{dL}$ ) and identified $F 7$ genetic lesions in combination with almost all pro- and anti-coagulant factors 
were found in 17 patients (33\%). Genetic defects in $F 7$ were detected in the homo-, hetero-, or compound heterozygous state. The clinical symptoms were depicted with different severity. In majority of patients, mild/moderate bleeding events including epistaxis, cutaneous bleeding, oral bleeding, bleeding after surgery or trauma, and menorrhagia were registered. In female patients, menorrhagia was the most common symptom.

Table 1. Laboratory and genetic features of the familial multiple coagulation factor deficiencies (FMCFDs) arising from single, co-inherited clotting factor genetic defect. FMCFDs due to deficiency (a) of two pro-coagulant factors, (b) two/three pro- and anti-coagulant factors, (c) two anti-coagulant factors ( $\mathrm{m}=$ male, $\mathrm{f}=$ female), $\mathrm{IU} / \mathrm{dL}=$ international units $/$ deciliter, $\mathrm{mg} / \mathrm{dL}=$ milligram/deciliter. Novel variants are bold.

\begin{tabular}{|c|c|c|c|c|c|c|}
\hline Patient & Sex & Gene & Nucleotide Position & Aminoacid Exchange & Protein & $\begin{array}{l}\text { Laboratory } \\
\text { Parameter }\end{array}$ \\
\hline \multicolumn{7}{|c|}{ (a) pro-coagulant factor deficiencies } \\
\hline \multirow{2}{*}{ P1 } & \multirow{2}{*}{$\mathrm{m}$} & $F 7$ & c. $[547 \mathrm{G}>\mathrm{A}] ;[=]$ & p.(Asp183Asn) & FVII & $54 \mathrm{IU} / \mathrm{dL}$ \\
\hline & & $F 2$ & c. $[1771 \mathrm{G}>\mathrm{A}] ;[=]$ & p.(Gly591Ser) & Prothrombin & $38 \mathrm{IU} / \mathrm{dL}$ \\
\hline \multirow{2}{*}{$\mathrm{P} 2$} & \multirow{2}{*}{$\mathrm{f}$} & $F 7$ & c. $[1061 C>T] ;[=]$ & p.(Ala354Val) & FVII & $58 \mathrm{IU} / \mathrm{dL}$ \\
\hline & & F5 & c. $[6230 \mathrm{~T}>\mathrm{C}] ;[=]$ & p.(Ile2077Thr) & $\mathrm{FV}$ & $31 \mathrm{IU} / \mathrm{dL}$ \\
\hline \multirow{2}{*}{ P3 } & \multirow{2}{*}{$\mathrm{m}$} & $F 7$ & c. $[215 C>G] ;[=]$ & p.(Ser72Cys) & FVII & $29 \mathrm{IU} / \mathrm{dL}$ \\
\hline & & F5 & c. $[4900 \mathrm{C}>\mathrm{T}] ;[=]$ & p.(Arg1634Ter) & $\mathrm{FV}$ & $48 \mathrm{IU} / \mathrm{dL}$ \\
\hline \multirow{2}{*}{$\mathrm{P} 4$} & \multirow{2}{*}{$\mathrm{m}$} & $F 7$ & c. $[796 \mathrm{G}>\mathrm{A}] ;[=]$ & p.(Ala266Thr) & FVII & $27 \mathrm{IU} / \mathrm{dL}$ \\
\hline & & F5 & c.[585_586insA];[=] & p.(Gly196Argfs) & FV & $42 \mathrm{IU} / \mathrm{dL}$ \\
\hline \multirow[b]{2}{*}{ P5 } & \multirow[b]{2}{*}{$\mathrm{m}$} & & [Large del exons 1-9];[=] & & & \\
\hline & & F10 & $\begin{array}{c}\text { c. }[1147 \mathrm{C}>\mathrm{T}] ;[\text { Large del exons } \\
1-6]\end{array}$ & p.(Pro383Ser) & FXII & $2 \mathrm{IU} / \mathrm{dL}$ \\
\hline \multirow{2}{*}{ P6 } & \multirow[b]{2}{*}{$\mathrm{m}$} & $F 7$ & c.[65-3C>T];[Large del exons & - & FVII & $32 \mathrm{IU} / \mathrm{dL}$ \\
\hline & & F10 & $\begin{array}{c}4-9] \\
\text { c.[Large del exon 1];[=] }\end{array}$ & - & FX & $36 \mathrm{IU} / \mathrm{dL}$ \\
\hline \multirow{2}{*}{ P7 } & \multirow{2}{*}{$\mathrm{f}$} & $F 7$ & c. $[1061 \mathrm{C}>\mathrm{T}(;) 1391 \mathrm{delC}] ;[=]$ & p.[(Ala354Val(;)Pro464Hisfs)] & FVII & $39 \mathrm{IU} / \mathrm{dL}$ \\
\hline & & F10 & c. $[706 \mathrm{G}>\mathrm{A}] ;[=]$ & p.(Val236 Met) & $\mathrm{FX}$ & $49 \mathrm{IU} / \mathrm{dL}$ \\
\hline \multirow{2}{*}{ P8 } & \multirow{2}{*}{$\mathrm{f}$} & $F 7$ & c. $[291+1 G>A] ;[=]$ & - & FVII & $41 \mathrm{IU} / \mathrm{dL}$ \\
\hline & & $F 10$ & c. $[301 \mathrm{~T}>\mathrm{C}] ;[=]$ & p.(Cys101Arg) & $\mathrm{FX}$ & $48 \mathrm{IU} / \mathrm{dL}$ \\
\hline \multirow{2}{*}{ P9 } & \multirow{2}{*}{$\mathrm{f}$} & $F 7$ & c. $[1072 \mathrm{~A}>\mathrm{G}] ;[=]$ & p.(Met358Val) & FVII & $51 \mathrm{IU} / \mathrm{dL}$ \\
\hline & & F10 & c. $[424 \mathrm{G}>\mathrm{A}] ;[=]$ & p.(Glu142Lys) & FX & $47 \mathrm{IU} / \mathrm{dL}$ \\
\hline \multirow{2}{*}{ P10 } & \multirow{2}{*}{$\mathrm{m}$} & $F 7$ & c. $[-55 \mathrm{C}>\mathrm{T}] ;[=]$ & - & FVII & $51 \mathrm{IU} / \mathrm{dL}$ \\
\hline & & $F 10$ & c. $[1107 \mathrm{G}>\mathrm{T}] ;[1108 \mathrm{~A}>\mathrm{T}]$ & p.[(Glu369Asp(;)Lys370Ter)] & FX & $25 \mathrm{IU} / \mathrm{dL}$ \\
\hline \multirow{2}{*}{ P11 } & \multirow[b]{2}{*}{$\mathrm{f}$} & $F 7$ & c. [589A >G];[=] & p.(Lys197Glu) & FVII & $49 \mathrm{IU} / \mathrm{dL}$ \\
\hline & & $F 10$ & c. $[424 \mathrm{G}>\mathrm{A}] ;[=]$ & p.(Glu142Lys) & $\mathrm{FX}$ & $60 \mathrm{IU} / \mathrm{dL}$ \\
\hline \multirow{2}{*}{ P12 } & \multirow{2}{*}{$\mathrm{f}$} & $F 7$ & c. $[1061 \mathrm{C}>\mathrm{T}] ;[=]$ & p.(Ala354Val) & FVII & $35 \mathrm{IU} / \mathrm{dL}$ \\
\hline & & $F G A$ & c. $[103 \mathrm{C}>\mathrm{T}] ;[=]$ & p.(Arg35Cys) & Fibrinogen & $70 \mathrm{mg} / \mathrm{dL}$ \\
\hline \multirow{2}{*}{ P13 } & \multirow{2}{*}{$\mathrm{m}$} & $F 7$ & c. $[479 A>G] ;[=]$ & p.(Gln160Arg) & FVII & $33 \mathrm{IU} / \mathrm{dL}$ \\
\hline & & $F G G$ & c. $[(323 C>G] ;[=]$ & p.(Ala108Gly) & Fibrinogen & $146 \mathrm{mg} / \mathrm{dL}$ \\
\hline & & $F 7$ & c. [817_831del];[=] & p.(Lys273_Asp277del) & FVII & $66 \mathrm{IU} / \mathrm{dL}$ \\
\hline P14 & $\mathrm{f}$ & $V W F$ & c. $[3518 \mathrm{G}>\mathrm{T}] ;[=]$ & p.(Cys1173Phe) & VWF (Act./Ag) & $(22 / 5) \mathrm{IU} / \mathrm{dL}$ \\
\hline & & $F 10$ & c. $[1333 \mathrm{C}>\mathrm{G}] ;[=]$ & p.(Arg445Gly) & FX & $55 \mathrm{IU} / \mathrm{dL}$ \\
\hline P15 & $\mathrm{f}$ & $F 2$ & c. $[940 \mathrm{C}>\mathrm{T}] ;[=]$ & p.(Arg314Cys) & Prothrombin & $65 \mathrm{IU} / \mathrm{dL}$ \\
\hline & & $F 10$ & c. $[1147 \mathrm{C}>\mathrm{T}] ;[=]$ & p.(Pro383Ser) & FX & $42 \mathrm{IU} / \mathrm{dL}$ \\
\hline P16 & $\mathrm{f}$ & $F 2$ & c. $[940 \mathrm{C}>\mathrm{T}] ;[=]$ & p.(Arg314Cys) & Prothrombin & $54 \mathrm{IU} / \mathrm{dL}$ \\
\hline & & $F 10$ & c. $[1277 \mathrm{G}>\mathrm{A}] ;[=]$ & p.(Arg426His) & FX & $50 \mathrm{IU} / \mathrm{dL}$ \\
\hline P17 & $\mathrm{f}$ & F5 & c. $[5844 \mathrm{G}>\mathrm{C}] ;[=]$ & p.(Trp1948Cys) & FV & $50 \mathrm{IU} / \mathrm{dL}$ \\
\hline & & $F 10$ & c. $[1097 \mathrm{G}>\mathrm{A}] ;[=]$ & p.(Arg366His) & FX & $45 \mathrm{IU} / \mathrm{dL}$ \\
\hline P18 & $\mathrm{m}$ & $F 13 B$ & c. $[1342 \mathrm{C}>\mathrm{T}] ;[=]$ & p.(Pro448Ser) & FXIII & $41 \mathrm{IU} / \mathrm{dL}$ \\
\hline
\end{tabular}


Table 1. Cont.

\begin{tabular}{|c|c|c|c|c|c|c|}
\hline Patient & Sex & Gene & Nucleotide Position & Aminoacid Exchange & Protein & $\begin{array}{l}\text { Laboratory } \\
\text { Parameter }\end{array}$ \\
\hline \multirow{2}{*}{ P19 } & \multirow{2}{*}{$\mathrm{m}$} & $F 10$ & c. $[424 \mathrm{G}>\mathrm{A}] ;[=]$ & p.(Glu142Lys) & FX & $64 \mathrm{IU} / \mathrm{dL}$ \\
\hline & & $F G G$ & c. $[323 \mathrm{C}>\mathrm{G}] ;[=]$ & p.(Ala108Gly) & Fibrinogen & $150 \mathrm{mg} / \mathrm{dL}$ \\
\hline \multirow{2}{*}{$\mathrm{P} 20$} & \multirow{2}{*}{$\mathrm{m}$} & F11 & c. $[901 \mathrm{~T}>\mathrm{C}] ;[=]$ & p.(Phe301Leu) & FXI & $40 \mathrm{IU} / \mathrm{dL}$ \\
\hline & & $F 2$ & c. $[604 \mathrm{C}>\mathrm{T}] ;[=]$ & p.(Gln202Ter) & Prothrombin & $51 \mathrm{IU} / \mathrm{dL}$ \\
\hline \multirow{2}{*}{$\mathrm{P} 21$} & \multirow{2}{*}{$\mathrm{m}$} & $F 11$ & c. $[1047 \mathrm{G}>\mathrm{C}] ;[=]$ & p.(Lys349Asn) & FXI & $53 \mathrm{IU} / \mathrm{dL}$ \\
\hline & & $F 13 A 1$ & c. $[233 \mathrm{G}>\mathrm{A}] ;[233 \mathrm{G}>\mathrm{A}]$ & p.(Arg78His) & FXIII & $3 \mathrm{IU} / \mathrm{dL}$ \\
\hline \multirow{2}{*}{$\mathrm{P} 22$} & \multirow{2}{*}{$\mathrm{m}$} & $F 11$ & c. $[1724 \mathrm{C}>\mathrm{T}] ;[=]$ & p.(Ser575Leu) & FXI & $42 \mathrm{IU} / \mathrm{dL}$ \\
\hline & & FGG & c. $[323 \mathrm{C}>\mathrm{G}] ;[=]$ & p.(Ala108Gly) & Fibrinogen & $140 \mathrm{mg} / \mathrm{dL}$ \\
\hline \multirow{2}{*}{$\mathrm{P} 23$} & \multirow{2}{*}{$\mathrm{f}$} & $F 11$ & c. [1443delT];[=] & p.(Ile481Metfs) & FXI & $45 \mathrm{IU} / \mathrm{dL}$ \\
\hline & & $V W F$ & c. $[3296 \mathrm{G}>\mathrm{A}] ;[=]$ & p.(Cys1099Tyr) & VWF (Act./Ag) & $\begin{array}{l}(32 / 25) \\
\mathrm{IU} / \mathrm{dL}\end{array}$ \\
\hline \multirow{2}{*}{$\mathrm{P} 24$} & \multirow{2}{*}{$\mathrm{m}$} & F11 & c. $[419 \mathrm{G}>\mathrm{A}] ;[=]$ & p.(Cys140Tyr) & FXI & $51 \mathrm{IU} / \mathrm{dL}$ \\
\hline & & $V W F$ & c. $[5828 \mathrm{G}>\mathrm{A}] ;[=]$ & p.(Arg1943His) & VWF (Act./Ag) & $\begin{array}{l}(57 / 41) \\
\mathrm{IU} / \mathrm{dL}\end{array}$ \\
\hline P25 & $\mathrm{f}$ & $\begin{array}{l}\text { FGG } \\
\text { F5 }\end{array}$ & $\begin{array}{c}\text { c. }[323 C>G] ;[=] \\
\text { c. }\left[6528+1 \_6528+4\right. \\
\text { delGTAG];[=] }\end{array}$ & $\begin{array}{c}\text { p.(Ala108Gly) } \\
-\end{array}$ & $\begin{array}{c}\text { Fibrinogen } \\
\text { FV }\end{array}$ & $\begin{array}{c}160 \mathrm{mg} / \mathrm{dL} \\
35 \mathrm{IU} / \mathrm{dL}\end{array}$ \\
\hline \multirow{2}{*}{$\mathrm{P} 26$} & \multirow{2}{*}{$\mathrm{f}$} & $F G B$ & c. $[794 \mathrm{C}>\mathrm{T}] ;[=]$ & p.(Pro265Leu) & Fibrinogen & $233 \mathrm{mg} / \mathrm{dL}$ \\
\hline & & $V W F$ & c. $[4751 \mathrm{~A}>\mathrm{G}] ;[=]$ & p.(Tyr1584Cys) & $\begin{array}{l}\text { VVWF } \\
\text { (Act./Ag) }\end{array}$ & $\begin{array}{l}(61 / 38) \\
\mathrm{IU} / \mathrm{dL}\end{array}$ \\
\hline \multirow{2}{*}{$\mathrm{P} 27$} & \multirow{2}{*}{$\mathrm{f}$} & $V W F$ & c. $[4094 \mathrm{~T}>C] ;[=]$ & p.(Leu1365Pro) & VWF (Act./Ag) & $(10 / 16)$ \\
\hline & & F5 & c. $[1669 \mathrm{~T}>C] ;[=]$ & p.(Trp557Arg) & $\mathrm{FV}$ & $\begin{array}{c}\mathrm{IU} / \mathrm{dL} \\
41 \mathrm{IU} / \mathrm{dL}\end{array}$ \\
\hline \multirow{2}{*}{$\mathrm{P} 28$} & \multirow{2}{*}{$\mathrm{m}$} & F8 & intron 1 inversion & - & FVIII & $<1 \mathrm{IU} / \mathrm{dL}$ \\
\hline & & F9 & c. $[835 \mathrm{G}>\mathrm{A}] ;[0]$ & p.(Ala279Thr) & FIX & $10 \mathrm{IU} / \mathrm{dL}$ \\
\hline \multirow{2}{*}{ P29 } & \multirow{2}{*}{$\mathrm{m}$} & F8 & c. $[7030 \mathrm{G}>\mathrm{A}] ;[0]$ & p.(Gly2344Ser) & FVIII & $<1 \mathrm{IU} / \mathrm{dL}$ \\
\hline & & FGG & c. $[323 C>G] ;[=]$ & p.(Ala108Gly) & Fibrinogen & $124 \mathrm{mg} / \mathrm{dL}$ \\
\hline \multirow{2}{*}{ P30 } & & F8 & c. $[1978 \mathrm{~A}>\mathrm{C}] ;[0]$ & p.(Ser660Arg) & FVIII & $<1 \mathrm{IU} / \mathrm{dL}$ \\
\hline & $\mathrm{m}$ & FGG & c. $[323 \mathrm{C}>\mathrm{G}] ;[=]$ & p.(Ala108Gly) & Fibrinogen & $101 \mathrm{mg} / \mathrm{dL}$ \\
\hline & & F8 & intron 22 inversion & - & FVIII & $<1 \mathrm{IU} / \mathrm{dL}$ \\
\hline P31 & $\mathrm{m}$ & $F G B$ & c. $[1433 \mathrm{G}>\mathrm{A}] ;[=]$ & p.(Arg478Lys) & Fibrinogen & $110 \mathrm{mg} / \mathrm{dL}$ \\
\hline & & $F 9$ & c. $[676 \mathrm{C}>\mathrm{T}] ;[0]$ & p.(Arg226Trp) & & $<1 \mathrm{IU} / \mathrm{dL}$ \\
\hline P32 & $\mathrm{m}$ & $F G B$ & c.[959-7_959-4delCTTTT];[=] & - & $\begin{array}{l}\text { FIX } \\
\text { Fibrinogen }\end{array}$ & $120 \mathrm{mo} / \mathrm{dL}$ \\
\hline & & FGG & c. $[323 C>G] ;[=]$ & p.(Ala108Gly) & & \\
\hline & & F8 & c. $[1293 G>T] ;[0]$ & p.(Leu431Phe) & FVIII & $3 \mathrm{IU} / \mathrm{dL}$ \\
\hline P33 & $\mathrm{m}$ & $F 7$ & c. $[1061 C>T] ;[=]$ & p.(Ala354Val) & FVII & $29 \mathrm{IU} / \mathrm{dL}$ \\
\hline & & F8 & c. [5398C >T];[0] & p.(Arg1800Cys) & FVIII & $4 \mathrm{IU} / \mathrm{dL}$ \\
\hline P34 & $\mathrm{m}$ & $V W F$ & c. $[6187 \mathrm{C}>\mathrm{T}] ;[=]$ & p.(Pro2063Ser) & VWF (Act./Ag) & $(43 / 30)$ \\
\hline & & & & & & IU/dL \\
\hline & & $F 8$ & c. [5793T>A];[0] & p.(Phe1931Leu) & FVIII & $46 \mathrm{IU} / \mathrm{dL}$ \\
\hline P35 & $\mathrm{m}$ & $F 11$ & c. $[1075 \mathrm{~A}>\mathrm{G}] ;[=]$ & p.(Ile359Val) & FXI & $41 \mathrm{IU} / \mathrm{dL}$ \\
\hline P36 & $m$ & $F 8$ & c. $[6325 \mathrm{C}>\mathrm{T}] ;[0]$ & p.(Arg2190Cys) & FVIII & $14 \mathrm{IU} / \mathrm{dL}$ \\
\hline 130 & $\mathrm{~m}$ & $F 11$ & c. $[1540 \mathrm{~T}>\mathrm{C}] ;[=]$ & p.(Cys514Arg) & FXI & $30 \mathrm{IU} / \mathrm{dL}$ \\
\hline P37 & $\mathrm{m}$ & F8 & c. $[752 \mathrm{~A}>\mathrm{C}] ;[0]$ & p.(His251Pro) & FVIII & $28 \mathrm{IU} / \mathrm{dL}$ \\
\hline 137 & $\mathrm{~m}$ & $F 13 A 1$ & c. $[547 \mathrm{~A}>\mathrm{G}] ;[=]$ & p.(Ile183 Val) & FXIII & $38 \mathrm{IU} / \mathrm{dL}$ \\
\hline P38 & $m$ & $F 8$ & c. $[121 \mathrm{G}>\mathrm{T}] ;[0]$ & p.(Gly41Cys) & FVIII & $6 \mathrm{IU} / \mathrm{dL}$ \\
\hline P38 & $\mathrm{m}$ & $F G G$ & c. $[323 \mathrm{C}>\mathrm{G}] ;[=]$ & p.(Ala108Gly) & Fibrinogen & $170 \mathrm{mg} / \mathrm{dL}$ \\
\hline & & F8 & c.[1481T>C];[0] & p.(Ile494Thr) & FVIII & $13 \mathrm{IU} / \mathrm{dL}$ \\
\hline P39 & $\mathrm{m}$ & $V W F$ & c. [4636delG];[=] & p.(Val1546Ter) & VWF (Act./Ag) & $\begin{array}{l}(10 / 15) \\
\mathrm{IU} / \mathrm{dL}\end{array}$ \\
\hline P40 & $\mathrm{m}$ & F8 & c.[Large dup exon 6];[0] & - & FVIII & $26 \mathrm{IU} / \mathrm{dL}$ \\
\hline 140 & $\mathrm{~m}$ & F7 & c. $[847 \mathrm{C}>\mathrm{T}] ;[=]$ & p.(Arg283Trp) & FVII & $40 \mathrm{IU} / \mathrm{dL}$ \\
\hline
\end{tabular}


Table 1. Cont.

\begin{tabular}{|c|c|c|c|c|c|c|}
\hline Patient & Sex & Gene & Nucleotide Position & Aminoacid Exchange & Protein & $\begin{array}{l}\text { Laboratory } \\
\text { Parameter }\end{array}$ \\
\hline \multirow{2}{*}{ P41 } & \multirow{2}{*}{$\mathrm{f}$} & F8 & c. $[1648 \mathrm{C}>\mathrm{T}] ;[=]$ & p.(Arg550Cys) & FVIII & $30 \mathrm{IU} / \mathrm{dL}$ \\
\hline & & $F 7$ & c. $[509 \mathrm{G}>\mathrm{A}] ;[=]$ & p.(Arg170His) & FVII & $15 \mathrm{IU} / \mathrm{dL}$ \\
\hline \multirow{2}{*}{ P42 } & \multirow{2}{*}{$\mathrm{f}$} & F8 & c. $[761 \mathrm{~A}>\mathrm{G}] ;[=]$ & p.(Asn254Ser) & FVIII & $17 \mathrm{IU} / \mathrm{dL}$ \\
\hline & & F10 & c. $[1237 \mathrm{G}>\mathrm{A}] ;[=]$ & p.(Asp413Asn) & FX & $14 \mathrm{IU} / \mathrm{dL}$ \\
\hline \multirow{2}{*}{$\mathrm{P} 43$} & \multirow{2}{*}{$\mathrm{f}$} & F8 & c. $[1971 C>G] ;[=]$ & p.(Tyr657Ter) & FVIII & $19 \mathrm{IU} / \mathrm{dL}$ \\
\hline & & $V W F$ & c. $[4751 \mathrm{~A}>\mathrm{G}] ;[=]$ & p.(Tyr1584Cys) & VWF (Act./Ag) & NA \\
\hline \multirow{2}{*}{$\mathrm{P} 44$} & \multirow{2}{*}{$\mathrm{f}$} & \multirow{2}{*}{\multicolumn{2}{|c|}{$\begin{array}{l}\text { c. }[871 \mathrm{G}>\mathrm{A}] ;[=] \\
\text { c. }[5278 \mathrm{G}>\mathrm{A}] ;[=]\end{array}$}} & \multirow{2}{*}{$\begin{array}{l}\text { p.(Glu291Lys) } \\
\text { p.(Val1760Ile) }\end{array}$} & \multirow{2}{*}{$\begin{array}{c}\text { FIX } \\
\text { VWF (Act./Ag) }\end{array}$} & \multirow{2}{*}{$\begin{array}{c}58 \mathrm{IU} / \mathrm{dL} \\
(40 / 35) \\
\mathrm{IU} / \mathrm{dL}\end{array}$} \\
\hline & & & & & & \\
\hline \multicolumn{7}{|c|}{ (b) pro-and anti- coagulant factor deficiencies } \\
\hline \multirow{2}{*}{ P45 } & \multirow{2}{*}{$\mathrm{f}$} & F9 & c. $[87 \mathrm{~A}>\mathrm{G}] ;[=]$ & p. $(=)$ & FIX & $22 \mathrm{IU} / \mathrm{dL}$ \\
\hline & & SERPINC1 & c. $[235 \mathrm{C}>\mathrm{T}] ;[=]$ & p.(Arg79Cys) & Antithormbin & $49 \mathrm{IU} / \mathrm{dL}$ \\
\hline \multirow{3}{*}{$\mathrm{P} 46$} & \multirow{3}{*}{$\mathrm{f}$} & $F 7$ & c. $[911 C>\mathrm{T}] ;[=]$ & p.(Ala304Val) & FVII & $51 \mathrm{IU} / \mathrm{dL}$ \\
\hline & & PROC & c. $[124 \mathrm{C}>\mathrm{T}] ;[425 \mathrm{~T}>\mathrm{C}]$ & p.[(Arg42Cys(;)Leu142Pro)] & Protein C & $14 \mathrm{IU} / \mathrm{dL}$ \\
\hline & & PROS1 & c. $[1501 \mathrm{~T}>\mathrm{C}] ;[=]$ & p.(Ser501Pro) & Free Protein S & $48 \mathrm{IU} / \mathrm{dL}$ \\
\hline \multirow{2}{*}{ P47 } & \multirow{2}{*}{$\mathrm{m}$} & $F G G$ & c. $[323 C>G] ;[=]$ & p.(Ala108Gly) & Fibrinogen & $130 \mathrm{mg} / \mathrm{dL}$ \\
\hline & & PROC & c. $[1267 \mathrm{G}>\mathrm{A}] ;[=]$ & p.(Gly423Ser) & Protein C & $68 \mathrm{IU} / \mathrm{dL}$ \\
\hline \multirow{2}{*}{$\mathrm{P} 48$} & \multirow{2}{*}{$\mathrm{f}$} & F11 & c. $[943 \mathrm{G}>\mathrm{A}] ;[=]$ & p.(Glu315Lys) & FXI & $43 \mathrm{IU} / \mathrm{dL}$ \\
\hline & & PROS1 & c. $[1501 \mathrm{~T}>\mathrm{C}] ;[=]$ & p.(Ser501Pro) & Free Protein S & $50 \mathrm{I} \mathrm{U} / \mathrm{dL}$ \\
\hline \multirow{2}{*}{ P49 } & \multirow[b]{2}{*}{$\mathrm{f}$} & $V W F$ & [Large del exon 6];[Large del & & VWF (Act./Ag) & $(1 / 1) \mathrm{IU} / \mathrm{dL}$ \\
\hline & & SERPINC1 & $\begin{array}{c}\text { exon 6] } \\
\text { c. }[133 \mathrm{C}>\mathrm{T}] ;[=]\end{array}$ & p.(Arg45Trp) & Antithormbin & $62 \mathrm{IU} / \mathrm{dL}$ \\
\hline \multirow{2}{*}{ P50 } & \multirow{2}{*}{$\mathrm{f}$} & F11 & c.[1443delT];[=] & p.(Ile481Metfs) & FXI & $42 \mathrm{IU} / \mathrm{dL}$ \\
\hline & & SERPINC1 & c. $[805 \mathrm{G}>\mathrm{A}] ;[=]$ & p.(Glu269Lys) & Antithormbin & $62 \mathrm{IU} / \mathrm{dL}$ \\
\hline \multicolumn{7}{|c|}{ (c) anti-coagulant factor deficiencies } \\
\hline \multirow{2}{*}{ P51 } & \multirow{2}{*}{$\mathrm{f}$} & PROS1 & c. $[119 \mathrm{G}>\mathrm{A}] ;[=]$ & p.(Arg40His) & Free Protein S & $57 \mathrm{IU} / \mathrm{dL}$ \\
\hline & & PROC & c. $[322 \mathrm{C}>\mathrm{A}] ;[\mathrm{[}=]$ & p.(His108Asn) & Protein C & $42 \mathrm{IU} / \mathrm{dL}$ \\
\hline \multirow{2}{*}{ P52 } & \multirow[b]{2}{*}{$\mathrm{m}$} & PROS1 & c. $[1871-2 A>G] ;[=]$ & - & Free Protein S & $30 \mathrm{IU} / \mathrm{dL}$ \\
\hline & & SERPINC1 & c. $[391 \mathrm{C}>\mathrm{T}] ;[=]$ & p.(Leu131Phe) & Antithormbin & $72 \mathrm{IU} / \mathrm{dL}$ \\
\hline
\end{tabular}

In the case of combined FVII and FX deficiency (P5), two genetic alterations were identified in the compound heterozygous state contributing to the low FX residual level and severe clinical presentation. The patient presented severe bleeding episodes (hemarthrosis in the first year of life).

FMCFDs with hemophilia A and B in combination with other coagulation factor disorders represent the second most common combined deficiency in our cohort. In males, the FVIII residual activities, as well as the severity of clinical symptoms were strongly correlated to the $F 8$ genetic defect. In severe cases, although hemophilia A was combined with other bleeding factor deficiencies, the genetic alterations in the second gene ( $F G B$ or FGG (P29, P30, P31), F9 (P28)) did not change the severity of the bleeding episodes. Interestingly, in cases where the underlying $F 8$ genetic variant leads to mild/moderate hemophilia combined with genetic defects in other genes such as VWF (P34, P39) and F11 (P35, P36), bleeding diathesis presented a stronger phenotype compared to the singlegene deficiency. Coincidental inheritance of von Willebrand disease (VWD) with other coagulation factor deficiencies (FV/FVII/FVIII/FIX/FXI/fibrinogen/anti-thrombin) was identified in 10 patients. In a majority of cases, the genetic defects were missense variants in the heterozygous state (except for P49). Seven patients exhibited symptoms of VWD Type 1, two of VWD Type 2 (A and M), and one of VWD Type 3 (Table 1a,b). 
In total, six patients showed co-inheritance of a pro- and anti-coagulant factor (Table 1b). Of interest is P49, a VWD Type 3 patient, due to the homozygous large deletion of exon 6. The index patient additionally exhibits an anti-thrombin deficiency, which was proved to be due to a genetic variation in SERPINC1. Clinically, this patient was presented with moderate to mild bleeding symptoms (epistaxis, seldom subcutaneous hematomas). A second family member, harboring the same genetic defect in VWF in homozygous state in the absence of anti-thrombin deficiency, suffered from severe bleeding symptoms (severe menorrhagia, hemarthrosis) requiring prophylactic treatment with VWF concentrate. In two patients, a co-inheritance of two anti-coagulant factor deficiencies was identified (Table 1c).

Of interest is also P46 with a co-inheritance of F7 with PROC and PROS1 defects, where low FVII levels could not attenuate the thrombotic tendency, and the patient was presented with recurrent, severe deep vein thrombosis (DVT). All other coincidentally inherited disorders such as FII/FX, FII/FXI, FV/FX, FIX/fibrinogen, FX/fibrinogen, FX/FXIII, protein $\mathrm{C}$ /protein $\mathrm{S}$, and protein $\mathrm{S} /$ antithrombin were extremely rare and were detected in one and seldom in more than one patient.

\subsubsection{Group II: Combined Deficiency Caused by a Single-Gene Defect}

This group involves patients with two monogenic FMCFDs-combined deficiency of FV and FVIII and VKDCF (vitamin K-dependent coagulation factor) deficiency (Table 2). In a single-gene disorder, patients with combined FV and FVIII deficiency are homozygous for loss-of-function pathogenic or likely pathogenic genetic variants in either LMAN1 or MCFD2 genes. Eighteen patients exhibited simultaneous reduction of plasma FV and FVIII activities (5\% to $49 \%$ IU/dL) and mild-to-moderate bleeding symptoms as cutaneous bleeding, epistaxis, bleeding after teeth extraction, surgery, menorrhagia, and increased wound bleeding. Eleven patients $(61 \%)$ carried homozygous null variants (nonsense, deletion/duplication, and splice-site) in LMAN1. In the remaining seven patients, genetic alterations were identified in MCFD2. In contrast to $L M A N 1$, the genetic variants found in MCFD2 included null and missense variants.

Table 2. Laboratory and genetic features of the FMCFDs arising from single genetic defects-combined deficiency of FV and FVIII and combined VKDCF deficiency $(\mathrm{m}=$ male, $\mathrm{f}=$ female); VKDCF-vitamin K-dependent clotting factors, $p .=$ synonymous variant, $\mathrm{NA}=$ not available due to vitamin $\mathrm{k}$ prophylaxis, $\mathrm{IU} / \mathrm{dL}=$ international units/deciliter.

\begin{tabular}{|c|c|c|c|c|c|}
\hline Patient & Sex & Gene & Nucleotide Position & Aminoacid Exchange & $\begin{array}{l}\text { Laboratory } \\
\text { Parameter }\end{array}$ \\
\hline P53 & $\mathrm{f}$ & LMAN1 & c. $[275 \mathrm{C}>\mathrm{G}] ;[275 \mathrm{C}>\mathrm{G}]$ & p.(Ser92Ter) & $\begin{array}{l}\text { FV } 25 \mathrm{IU} / \mathrm{dL} \\
\text { FVIII } 21 \mathrm{IU} / \mathrm{dL}\end{array}$ \\
\hline P54 & $\mathrm{m}$ & LMAN1 & c. $[904 \mathrm{~A}>\mathrm{T}] ;[904 \mathrm{~A}>\mathrm{T}]$ & p.(Lys302Ter) & $\begin{array}{r}\text { FV } 27 \mathrm{IU} / \mathrm{dL} \\
\mathrm{FVIII} 38 \mathrm{IU} / \mathrm{dL}\end{array}$ \\
\hline P55 & $\mathrm{m}$ & LMAN1 & c. $[313 \mathrm{G}>\mathrm{T}] ;[313 \mathrm{G}>\mathrm{T}]$ & p.(Glu105Ter) & $\begin{array}{l}\text { FV } 35 \mathrm{IU} / \mathrm{dL} \\
\text { FVIII } 16 \mathrm{IU} / \mathrm{dL}\end{array}$ \\
\hline P56 & $\mathrm{m}$ & LMAN1 & c. $[904 \mathrm{~A}>\mathrm{T}] ;[904 \mathrm{~A}>\mathrm{T}]$ & p.(Lys302Ter) & $\begin{array}{l}\text { FV } 14 \mathrm{IU} / \mathrm{dL} \\
\text { FVIII } 7 \mathrm{IU} / \mathrm{dL}\end{array}$ \\
\hline P57 & $\mathrm{m}$ & LMAN1 & c.[690delT];[690delT] & p.(Cys230Trpfs) & $\begin{array}{c}\text { FV } 8 \mathrm{IU} / \mathrm{dL} \\
\text { FVIII } 11 \mathrm{U} / \mathrm{dL}\end{array}$ \\
\hline P58 & $\mathrm{m}$ & LMAN1 & c.[839_840delA];[839_840delA] & p.(Ile281Phefs) & $\begin{array}{l}\text { FV } 23 \mathrm{IU} / \mathrm{dL} \\
\text { FVIII } 57 \mathrm{IU} / \mathrm{dL}\end{array}$ \\
\hline P59 & $\mathrm{m}$ & LMAN1 & c.[690delT];[690delT] & p.(Cys230Trpfs) & $\begin{array}{c}\text { FV } 9 \mathrm{IU} / \mathrm{dL} \\
\text { FVIII } 12 \mathrm{IU} / \mathrm{dL}\end{array}$ \\
\hline P60 & $\mathrm{m}$ & LMAN1 & c.[912dupA];[912dupA] & p.(Glu305Argfs) & $\begin{array}{l}\text { FV } 17 \mathrm{IU} / \mathrm{dL} \\
\text { FVIII } 31 \mathrm{IU} / \mathrm{dL}\end{array}$ \\
\hline P61 & $\mathrm{m}$ & LMAN1 & c. $[822 \mathrm{G}>\mathrm{A}] ;[822 \mathrm{G}>\mathrm{A}]$ & p. $(=)$ & $\begin{array}{l}\text { FV } 13 \mathrm{IU} / \mathrm{dL} \\
\text { FVIII } 17 \mathrm{IU} / \mathrm{dL}\end{array}$ \\
\hline P62 & $\mathrm{m}$ & LMAN1 & $\begin{array}{c}\text { c. }\left[822+33 \_822+34 \text { insGGTT]; }[822\right. \\
\text { +33_822+34insGGTT] }\end{array}$ & - & $\begin{array}{l}\text { FV } 49 \mathrm{IU} / \mathrm{dL} \\
\mathrm{FVIII} 40 \mathrm{IU} / \mathrm{dL}\end{array}$ \\
\hline
\end{tabular}


Table 2. Cont.

\begin{tabular}{|c|c|c|c|c|c|}
\hline Patient & Sex & Gene & Nucleotide Position & Aminoacid Exchange & $\begin{array}{l}\text { Laboratory } \\
\text { Parameter }\end{array}$ \\
\hline P63 & $\mathrm{m}$ & LMAN1 & $\begin{array}{c}\text { c.[151_163 } \\
\text { insdelTCGAAA];[151_163 } \\
\text { insdelTCGAAA] }\end{array}$ & p.(Ser51fs) & $\begin{array}{l}\text { FV } 31 \mathrm{IU} / \mathrm{dL} \\
\text { FVIII } 16 \mathrm{IU} / \mathrm{dL}\end{array}$ \\
\hline P64 & $\mathrm{f}$ & MCFD2 & c. $[407 \mathrm{~T}>\mathrm{C}] ;[407 \mathrm{~T}>\mathrm{C}]$ & p.(Ile136Thr) & $\begin{array}{c}\text { FV } 5 \mathrm{IU} / \mathrm{dL} \\
\text { FVIII } 21 \mathrm{IU} / \mathrm{dL}\end{array}$ \\
\hline P65 & $\mathrm{m}$ & MCFD2 & c. $[266 \mathrm{~A}>\mathrm{C}] ;[266 \mathrm{~A}>\mathrm{C}]$ & p.(Asp89Ala) & $\begin{array}{l}\text { FV } 16 \mathrm{IU} / \mathrm{dL} \\
\text { FVIII } 8 \mathrm{IU} / \mathrm{dL}\end{array}$ \\
\hline P66 & $\mathrm{m}$ & MCFD2 & c.[249delT];[249delT] & p.(Asp83Glufs) & $\begin{array}{c}\text { FV } 12 \mathrm{IU} / \mathrm{dL} \\
\text { FVIII } 10 \mathrm{IU} / \mathrm{dL}\end{array}$ \\
\hline P67 & $\mathrm{m}$ & MCFD2 & c.[249delT];[249delT] & p.(Asp83Glufs) & $\begin{array}{l}\text { FV } 10 \mathrm{IU} / \mathrm{dL} \\
\text { FVIII } 8 \mathrm{IU} / \mathrm{dL}\end{array}$ \\
\hline P68 & $\mathrm{m}$ & MCFD2 & c. $[309+1 G>A] ;[309+1 G>A]$ & - & $\begin{array}{c}\text { FV } 12 \mathrm{IU} / \mathrm{dL} \\
\text { FVIII } 11 \mathrm{IU} / \mathrm{dL}\end{array}$ \\
\hline P69 & $\mathrm{f}$ & MCFD2 & c. $[149+1 \mathrm{G}>\mathrm{A}] ;[149+1 \mathrm{G}>\mathrm{A}]$ & - & $\begin{array}{c}\text { FV } 7 \mathrm{U} / \mathrm{dL} \\
\text { FVIII } 10 \mathrm{IU} / \mathrm{dL}\end{array}$ \\
\hline P70 & $\mathrm{m}$ & MCFD2 & c.[91_112dup];[91_112dup] & p.(Met39Glnfs) & $\begin{array}{c}\text { FV } 9 \text { IU/dL } \\
\text { FVIII } 17 \text { IU/dL }\end{array}$ \\
\hline P71 & $\mathrm{f}$ & GGCX & c. $[944 \mathrm{G}>\mathrm{A}(;) 1454 \mathrm{G}>\mathrm{C}]$ & p.[(Trp315Ter(;)Arg485Pro)] & NA \\
\hline P72 & $\mathrm{f}$ & GGCX & c. $[417 \mathrm{~T}>\mathrm{G}(;) 1609+3 \mathrm{~A}>\mathrm{G}]$ & p.(Cys139Trp) & $\begin{array}{c}\text { FII:25 IU/dL, } \\
\text { FVII:34 IU/dL } \\
\text { FX:18 IU/dL }\end{array}$ \\
\hline P73 & $\mathrm{m}$ & GGCX & c. $[239 \mathrm{C}>\mathrm{T}(;) 1157 \mathrm{G}>\mathrm{T}(;) 1595 \mathrm{~T}>\mathrm{C}]$ & p.[(Pro80Leu(;)Gly386Val(;)Ile532Thr)] & NA \\
\hline
\end{tabular}

In our cohort, we identified three patients with combined VKDCF deficiency where the abnormal carboxylase function arises from defects in the gene encoding the $\gamma$-gGlutamyl carboxylase (GGCX) enzyme. In all three patients, the disorder arose from genetic variants in compound heterozygous state. The clinical symptoms varied widly from asymptomatic (P72) to intracerebral bleedings (P73).

\subsubsection{Group III: Combined Deficiency Arising from Cytogenetic Abnormalities}

In this group, we report nine patients who display combined deficiency of FVII and FX due to $13 q$ terminal deletion. The genes encoding these two proteins are mapped to 13q34. All cases were associated with subclinical deficiencies of factors VII and X where the residual plasma activity of each factor was reduced up to $50 \%$ of the normal values. The bleeding symptoms were mild, and mainly presented with cutaneous bleedings, epistaxis, oral cavity bleedings, and menorrhagia. All cases show intermediate levels of activity, suggesting heterozygosity (Table 3).

\subsection{Profile of Genetic Variants in FMCFDs}

Molecular analysis revealed 163 genetic variants in 82 patients with FMCFDs. The majority of genetic defects $(58.9 \%)$ were missense variants similar to their prevalence in the single-gene deficiency. Interestingly, the proportion of large deletions in comparison to that in the single-gene deficiencies was rather high $(9.2 \%)$, mostly owing to the cohort of nine patients with combined FVII/FX deficiency due to the distal deletion of chromosome 13. Small deletions/insertions, splice-site, and nonsense variants represent $15.3 \%, 7.4 \%$, and $6.7 \%$ of identified genetic variants in our cohort, respectively (Figure 1a). Splice-site genetic variants were mainly in intronic regions affecting either the donor or acceptor splicing site of the respective gene. In P61, the genetic alteration affected the last nucleotide of the exon 7 of the LMAN1 gene resulting in no amino acid change but abolishment 
of the splice-site. Except for the LMAN1 gene, where only null variants were detected, in all other genes, all types of genetic variants (nonsense, missense, deletion/duplication, and splice-site) were documented. In all, 85 unique genetic variants were already registered in the single-gene database as disease causative, and the remaining $33(28 \%)$ were novel.

Table 3. Laboratory and genetic features of the FMCFDs arising from cytogenetic abnormalities $\mathrm{IU} / \mathrm{dL}=$ international units $/$ deciliter $(\mathrm{m}=$ male, $\mathrm{f}=$ female $)$.

\begin{tabular}{|c|c|c|c|}
\hline Patient & Sex & Gene & $\begin{array}{l}\text { Laboratory } \\
\text { Parameter }\end{array}$ \\
\hline P74 & $\mathrm{f}$ & $\begin{array}{c}F 7 \\
F 10\end{array}$ & $\begin{array}{l}58 \mathrm{IU} / \mathrm{dL} \\
39 \mathrm{IU} / \mathrm{dL}\end{array}$ \\
\hline P75 & $\mathrm{m}$ & $\begin{array}{l}F 7 \\
F 10\end{array}$ & $\begin{array}{l}42 \mathrm{IU} / \mathrm{dL} \\
36 \mathrm{IU} / \mathrm{dL}\end{array}$ \\
\hline P76 & $\mathrm{f}$ & $\begin{array}{l}F 7 \\
F 10\end{array}$ & $\begin{array}{l}35 \mathrm{IU} / \mathrm{dL} \\
55 \mathrm{IU} / \mathrm{dL}\end{array}$ \\
\hline P77 & $\mathrm{f}$ & $\begin{array}{c}F 7 \\
F 10 \\
\end{array}$ & $\begin{array}{l}42 \mathrm{IU} / \mathrm{dL} \\
51 \mathrm{IU} / \mathrm{dL}\end{array}$ \\
\hline P78 & $\mathrm{m}$ & $\begin{array}{l}F 7 \\
F 10\end{array}$ & $\begin{array}{l}25 \mathrm{IU} / \mathrm{dL} \\
50 \mathrm{IU} / \mathrm{dL}\end{array}$ \\
\hline P79 & $\mathrm{f}$ & $\begin{array}{l}F 7 \\
F 10\end{array}$ & $\begin{array}{l}22 \mathrm{IU} / \mathrm{dL} \\
45 \mathrm{IU} / \mathrm{dL}\end{array}$ \\
\hline P80 & $\mathrm{f}$ & $\begin{array}{l}F 7 \\
F 10 \\
\end{array}$ & $\begin{array}{l}48 \mathrm{IU} / \mathrm{dL} \\
44 \mathrm{IU} / \mathrm{dL}\end{array}$ \\
\hline P81 & $\mathrm{f}$ & $\begin{array}{l}F 7 \\
F 10\end{array}$ & $\begin{array}{l}30 \mathrm{IU} / \mathrm{dL} \\
50 \mathrm{IU} / \mathrm{dL}\end{array}$ \\
\hline P82 & $\mathrm{f}$ & $\begin{array}{c}F 7 \\
F 10\end{array}$ & $\begin{array}{l}54 \mathrm{IU} / \mathrm{dL} \\
53 \mathrm{IU} / \mathrm{dL}\end{array}$ \\
\hline
\end{tabular}
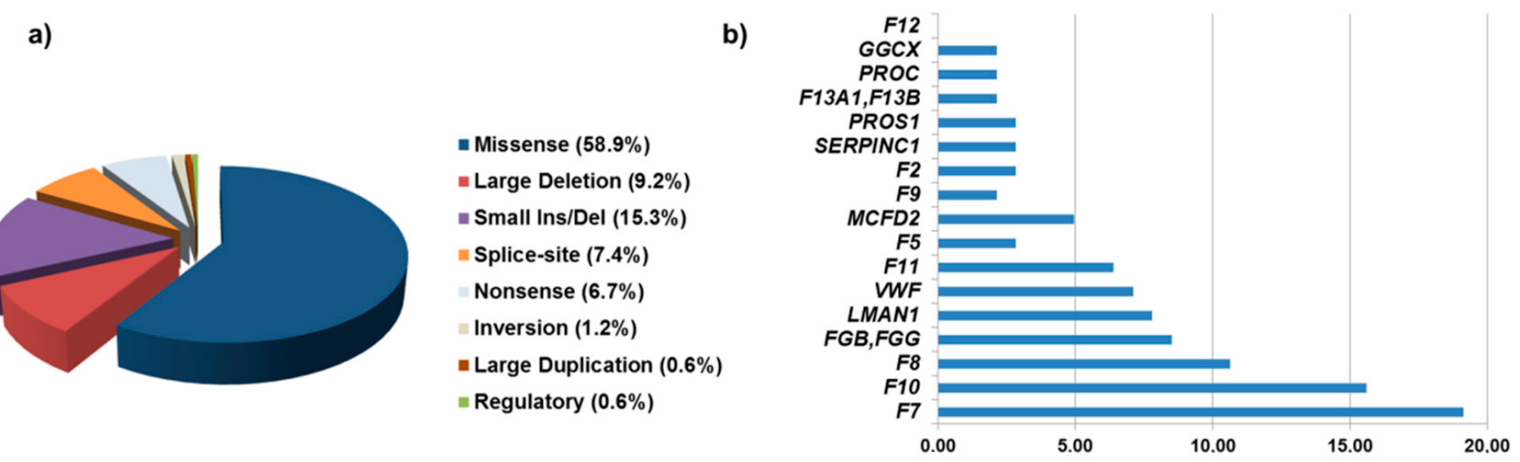

Figure 1. Genetic variants in patients with FMCFD. (a) Profile of genetic variants and (b) the prevalence of single-factor deficiencies in our study.

In cases with variants with uncertain significance, the causality of the genetic defect was confirmed either by segregation analysis or after exclusion of the identified variant in unaffected family members. In our cohort, 34 different combinations of single-gene deficiency were detected, where factor VII deficiency was frequently involved in combination (Figure 1b). Nearly all coagulation factors (except FXII), with different prevalence, were engaged in this interplay. Moreover, combinations of rather common monogenic disorders (hemophilia A, VWD, factor VII deficiency) were more prevalent than those involving combinations of rare monogenic disorders (factor V, factor II, factor XIII deficiency). 


\section{Discussion}

In this study, 82 patients with more than one inherited coagulation factor deficiency were investigated. Majority of our patients constitute the group of inheritance of two different affected genes, followed by FMCFDs due to single-gene defect. A small part represents a group of FMCFDs caused by partial deletion of chromosome 13. The great majority of these deficiencies were associated with bleeding disorders, while the association with thrombotic phenotype was rare.

Bearing in mind, that the rare bleeding disorders (RBDs) represent $3-5 \%$ of all inherited deficiencies of coagulation factors, the prevalence of FMCFDs is expected to be much less. Thus, FMCFDs are more sporadic and only isolated case reports are available $[23,28,29]$. As a consequence of the rarity of these deficiencies, the type and severity of symptoms, the underlying molecular defects, the interaction between the different coagulation factors, and the actual management of bleeding/thrombotic episodes are not well established. The current study presents genetic data on the largest collected and investigated cohort of patients with FMCFD.

Several studies have highlighted the complex biosynthetic pathway of single coagulation factors, which includes several post-translational modifications required for factor secretion and function. Alterations in genes that participate in the protein maturation process, produce and modulate rare coagulation deficiencies as a combined deficiency of coagulation FV and FVIII caused by pathogenic genetic variants in genes encoding proteins involved in the FV and FVIII intracellular transport (MCFD2 and LMAN1) and combined deficiency of all vitamin $\mathrm{K}$-dependent coagulation factors caused by pathogenic genetic variants in the gene coding for GGCX and VKORC1 [8,30,31].

The detected genetic defects in the LMAN1 gene accounting for the deficiency in all cases were null variants such as nonsense, small deletion/insertion, and splice-site (group II). Clinical symptoms were of a moderate to mild bleeding phenotype. Since its discovery, the combined FV and FVIII deficiency is the most common and well-studied FMCFD. Most described cases come from Mediterranean, Middle Eastern, and South Asian countries likely due to the prevalence of consanguineous marriages in these regions. Sixty percent of our patients had Middle Eastern origin, and all detected variants were in the homozygous state.

The prevalence of large deletions in our cohort was high mainly as a result of FMCFDs arising from cytogenetic abnormalities. This FMCFD was as a consequence of a deletion in the long arm of chromosome 13 involving both FVII- and FX-coding genes, which are known to be in the close vicinity [11,23,32]. Depending on the size of the deletion, other genes, except $F 7$ and $F 10$, could be affected. In such cases, the clinical phenotype could be presented with a large variability including abnormalities such as mental retardation, abnormal fascia or cardiovascular, and musculoskeletal and urogenital developmental anomalies. Such symptoms were not reported in any of our patients, suggesting that the deletion involves the very distal end of chromosome 13, affecting only F7 and F10 genes.

Analysis of data of involvement of the single-gene deficiency in FMCFDs showed that nearly all (except FXII) coagulation factors were engaged in this interplay with different prevalences. Moreover, combinations of rather common monogenic disorders (hemophilia A, VWD, factor VII deficiency) were more prevalent than those involving combinations of rare monogenic disorders (factor V, factor II, factor XIII deficiency). In our study, we described 34 different combinations of single-gene deficiency, where factor VII deficiency was frequently involved in combination. An explanation of this finding could be that factor VII deficiency is the most common deficiency $(1$ in 500,000) among all RBDs [33]. Additionally, FVII deficiency was involved in two groups of FMCFDs-FMCFDs arising from cytogenetic abnormalities and FMCFDs with co-inherited, individual clotting factor genetic defect.

In general, the clinical phenotype in patients with inherited coagulation disorders is related to the residual factor level in the plasma, where the gene variant is the main determinant of such levels. The large heterogeneity of the variant spectrum further provides 
complexity to the genotype-phenotype relationship. Genetic variants that interrupt protein production (deletion, insertion, splicing variant, nonsense variant) cause a more severe phenotype than non-null and missense variants. However, the relation between genotype and phenotype is not always clearly defined, as the severity of clinical symptoms is highly variable. Even among patients with the same genotype, additional acquired and genetic factors, i.e., functional polymorphisms could modulate the clinical expression of deficiencies. This genotype-phenotype correlation is even more difficult to establish in patients with FMCFDs. Some combinations may increase the severity of bleeding (coinheritance of two pro-coagulant genes) or of thrombotic events (co-inheritance of two anti-coagulant genes) whereas others may ameliorate the phenotype (co-inheritance of proand anti-coagulant genes). As most RBDs are inherited autosomal recessive, the zygosity status of the genetic defect strongly influences the clinical manifestation.

Further complexity in the genotype-phenotype relationship is represented by the delicate balance between coagulation and anticoagulation. Moreover, some coagulation factors, such as thrombin and FV, have both procoagulant and anticoagulant functions [34,35]. It is already documented that co-inheritance of prothrombotic FV Leiden and prothrombin variant (G20210A) mitigate the clinical course in patients with hemophilia [36-38]. In combined deficiency of VWF and antithrombin (P49 in our study), both abnormalities counterbalanced each other and brought out a mild hemorrhagic phenotype despite a severe coagulation plasma deficiency caused by the severe genetic defect (homozygous large deletion).

The presence of two clotting factor deficiencies still causes diagnostic and treatment difficulties. The primary diagnosis of most combined deficiencies is usually based on a clinical interpretation of bleeding symptoms as well as performance of standard functional clotting assays.

However, some currently available laboratory assays for coagulation-factor activity are not very sensitive [39]. Moreover, the evaluation of low levels of majority of factors is challenging, leading to false diagnosis or severity assignment [40]. In some cases, the prolonged aPTT can be wrongly interpreted as a monogenic coagulation-factor deficiency and be assumed that the coagulation pattern is due to only one defect. Molecular analysis may clarify some diagnostic uncertainties, but before the era of NGS, the genetic analyses were elaborate. In patients with FMCFD, the availability of NGS has an increasing importance as this technology provides enhanced opportunities simultaneously to detect genetic variants in a large number of genes. Moreover, the NGS technology enables the detection of $\mathrm{CNVs}$, providing a good tool for the detection of large deletions and duplications especially in cases where no MLPA is available. Furthermore, whole-genome or whole-exome sequencing approaches could be applied in an extended research approach to identify new mechanism or new genes involved in the pathogenesis of FMCFDs.

Although NGS facilitates the detection process of genetic, it is often challenging to interpret the causality of all identified genetic variants, especially in case of missense variants. Additionally, the majority of the reported missense variants are unique changes for a given patient, where only experimental studies and investigation of extended family members could contribute immensely to the clarification of the genotype-phenotype correlation. Achieving the correct diagnosis would allow better clinical decision making and disease management, i.e., the choice of treatment, disease prognosis, and family planning.

\section{Conclusions}

FMCFDs are heritable abnormalities of hemostasis with a low overall population frequency, rendering them typically orphan diseases. To avoid misdiagnosis, the combination of comprehensive screening of residual activities, molecular analysis, and family segregation analysis should be performed to achieve the correct diagnosis.

Author Contributions: Conceptualization, A.P. and J.O.; methodology, B.P. (Barbara Preisler), A.P., and B.P. (Behnaz Pezeshkpoor); software, B.P. (Barbara Preisler), A.P., and B.P. (Behnaz Pezeshkpoor); 
validation, B.P. (Barbara Preisler), A.P., and B.P. (Behnaz Pezeshkpoor); formal analysis, B.P. (Barbara Preisler), A.P., and B.P. (Behnaz Pezeshkpoor); investigation, A.B., R.F., B.Z., U.S. (Ute Scholz), H.R., B.K.-M., U.S. (Ursula Schmitt), A.R., S.U., H.-J.L., and M.O.; resources, B.P. (Barbara Preisler), A.P., and B.P. (Behnaz Pezeshkpoor); data curation, B.P. (Barbara Preisler), A.P., and B.P. (Behnaz Pezeshkpoor); writing—original draft preparation, B.P. (Barbara Preisler) and A.P.; writing-review and editing, B.P. (Behnaz Pezeshkpoor); visualization, B.P. (Barbara Preisler), A.P., and B.P. (Behnaz Pezeshkpoor); supervision, A.P.; project administration, B.P. (Barbara Preisler); funding acquisition, A.P., B.P. (Barbara Preisler), and J.O. All authors have read and agreed to the published version of the manuscript.

Funding: This research was funded by CSL Behring Germany.

Institutional Review Board Statement: The study was conducted according to the guidelines of the Declaration of Helsinki and approved by the Ethics Committee of University of Bonn (191/18, 17.08.2018).

Informed Consent Statement: Informed consent was obtained from all subjects involved in the study.

Data Availability Statement: Data is contained within the article.

Conflicts of Interest: The authors declare no conflict of interest.

\section{References}

1. Soff, G.A.; Levin, J. Familial multiple coagulation factor deficiencies. I. Review of the literature: Differentiation of single hereditary disorders associated with multiple factor deficiencies from coincidental concurrence of single factor deficiency states. Semin. Thromb. Hemost. 1981, 7, 112-148. [CrossRef]

2. Soff, G.A.; Levin, J.; Bell, W.R. Familial multiple coagulation factor deficiencies. II. Combined factor VIII, IX, and XI deficiency and combined factor IX and XI deficiency: Two previously uncharacterized familial multiple factor deficiency syndromes. Semin. Thromb. Hemost. 1981, 7, 149-169. [CrossRef]

3. Robson, P.J.; Mumford, A.D. Familial multiple coagulation factor deficiencies-Chance associations and distinct clinical disorders. Haemoph. Off. J. World Fed. Hemoph. 2009, 15, 11-19. [CrossRef]

4. O'Brien, S.H.; Ritchey, A.K.; Ragni, M.V. Combined clotting factor deficiencies: Experience at a single hemophilia treatment center. Haemoph. Off. J. World Fed. Hemoph. 2007, 13, 26-29. [CrossRef]

5. Guglielmone, H.A.; Bastos, L.; Jarchum, G.D.; Alvarez-Bollea, M.A. Recurrent superficial venous thrombophlebitis because of mutations in the protein $C$ and fibrinogen genes in a young Argentinian female. Blood Coagul. Fibrinolysis 2019, 30, 80-84. [CrossRef]

6. Naderi, M.; Tabibian, S.; Hosseini, M.S.; Alizadeh, S.; Hosseini, S.; Shamsizadeh, M.; Dorgalaleh, A. Congenital combined deficiency of coagulation factors: A study of seven patients. Blood Coagul. Fibrinolysis 2015, 26, 59-62. [CrossRef]

7. Zheng, C.L.; Zhang, B. Combined Deficiency of Coagulation Factors V and VIII: An Update. Semin. Thromb. Hemost. 2013, 39, 613-620. [CrossRef]

8. Spreafico, M.; Peyvandi, F. Combined Factor V and Factor VIII Deficiency. Semin. Thromb. Hemost. 2009, 35, 390-399. [CrossRef]

9. Rost, S.; Fregin, A.; Koch, D.; Compes, M.; Muller, C.R.; Oldenburg, J. Compound heterozygous mutations in the gamma-glutamyl carboxylase gene cause combined deficiency of all vitamin K-dependent blood coagulation factors. Brit. J. Haematol. 2004, 126, 546-549. [CrossRef]

10. Napolitano, M.; Mariani, G.; Lapecorella, M. Hereditary combined deficiency of the vitamin K-dependent clotting factors. Orphanet J. Rare Dis. 2010, 5, 21. [CrossRef]

11. Pfeiffer, R.A.; Ott, R.; Gilgenkrantz, S.; Alexandre, P. Deficiency of Coagulation Factor-Vii and Factor-X Associated with Deletion of a Chromosome 13 (Q34)—Evidence from 2 Cases with 46,Xy,T(13-Y)(Q11-Q34). Hum. Genet. 1982, 62, 358-360. [CrossRef]

12. Peyvandi, F.; Duga, S.; Akhavan, S.; Mannucci, P.M. Rare coagulation deficiencies. Haemoph. Off. J. World Fed. Hemoph. 2002, 8 , 308-321. [CrossRef]

13. Palla, R.; Peyvandi, F.; Shapiro, A.D. Rare bleeding disorders: Diagnosis and treatment. Blood 2015, 125, 2052-2061. [CrossRef]

14. Peyvandi, F.; Menegatti, M.; Palla, R. Rare Bleeding Disorders: Worldwide Efforts for Classification, Diagnosis, and Management. Semin. Thromb. Hemost. 2013, 39, 579-584.

15. Sivapalaratnam, S.; Collins, J.; Gomez, K. Diagnosis of inherited bleeding disorders in the genomic era. Br. J. Haematol. 2017, 179, 363-376. [CrossRef]

16. Ver Donck, F.; Downes, K.; Freson, K. Strengths and limitations of high-throughput sequencing for the diagnosis of inherited bleeding and platelet disorders. J. Thromb. Haemost. 2020. [CrossRef]

17. Prabhudesai, A.; Shanbhag, S.; Mirgal, D.; Kawankar, N.; Shetty, S. A de novo factor VIII mutation in a haemophilia B family leading to combined deficiency of factor VIII and IX. Haemoph. Off. J. World Fed. Hemoph. 2017, 23, e477-e479. [CrossRef]

18. Girolami, A.; Zanon, E.; Bertomoro, A.; Gavasso, S.; Fadin, M. Combined factor V and factor VII deficiency due to an independent segregation of the two defects. Clin. Appl. Thromb. Hemost. 1999, 5, 136-138. [CrossRef] 
19. Knoll, B.; Hach-Wunderle, V.; Rieger, S.; Haring, D.; Mannhalter, C. Combined occurrence of a heterozygous missense mutation in the protein $C$ gene and allelic exclusion of one protein $S$ allele leading to severe venous thrombosis. Thromb. Res. 2001, 103, 3-8. [CrossRef]

20. Zhang, B.; Spreafico, M.; Zheng, C.; Yang, A.; Platzer, P.; Callaghan, M.U.; Avci, Z.; Ozbek, N.; Mahlangu, J.; Haw, T.; et al. Genotype-phenotype correlation in combined deficiency of factor V and factor VIII. Blood 2008, 111, 5592-5600. [CrossRef]

21. Zhang, B.; McGee, B.; Yamaoka, J.S.; Guglielmone, H.; Downes, K.A.; Minoldo, S.; Jarchum, G.; Peyvandi, F.; De Bosch, N.B.; Ruiz-Saez, A.; et al. Combined deficiency of factor V and factor VIII is due to mutations in either LMAN1 or MCFD2. Blood 2006, 107, 1903-1907. [CrossRef]

22. Al Absi, H.S.; Abdullah, M.F. Congenital Combined Deficiency of the Vitamin K-dependent Clotting Factors (VKCFD): A Novel Gamma-glutamyl Carboxylase (GGCX) Mutation. J. Pediatr. Hematol. Oncol. 2019, 41, e224-e226. [CrossRef]

23. Pavlova, A.; Preisler, B.; Driesen, J.; de Moerloose, P.; Zieger, B.; Hütker, S.; Dengler, K.; Harbrecht, U.; Oldenburg, J. Congenital combined deficiency of coagulation factors VII and X-Different genetic mechanisms. Haemoph. Off. J. World Fed. Hemoph. 2015, 21, 386-391. [CrossRef]

24. Ivaškevičius, V.; Pezeshkpoor, B.; Biswas, A.; Goldmann, G.; Horneff, S.; Gimbutyte, M.; Malciute, L.; Jurgutis, R. Combined coagulation factor VIII and factor IX deficiency (CDF8F9) in a patient from Lithuania. Hamostaseologie 2016, 36, S29-S33.

25. Richards, S.; Aziz, N.; Bale, S.; Bick, D.; Das, S.; Gastier-Foster, J.; Grody, W.W.; Hegde, M.; Lyon, E.; Spector, E.; et al. Standards and guidelines for the interpretation of sequence variants: A joint consensus recommendation of the American College of Medical Genetics and Genomics and the Association for Molecular Pathology. Genet. Med. 2015, 17, 405-424. [CrossRef]

26. Stenson, P.D.; Mort, M.; Ball, E.V.; Shaw, K.; Phillips, A.; Cooper, D.N. The Human Gene Mutation Database: Building a comprehensive mutation repository for clinical and molecular genetics, diagnostic testing and personalized genomic medicine. Hum. Genet. 2014, 133, 1-9. [CrossRef]

27. Rehm, H.L.; Berg, J.S.; Brooks, L.D.; Bustamante, C.D.; Evans, J.P.; Landrum, M.J.; Ledbetter, D.H.; Maglott, D.R.; Martin, C.L.; Nussbaum, R.L.; et al. ClinGen-The Clinical Genome Resource. N. Engl. J. Med. 2015, 372, 2235-2242. [CrossRef]

28. Asatiani, E.; Kessler, C.M. Directors of the Comprehensive Hemophilia Treatment Centers in R, III. Multiple congenital coagulopathies co-expressed with Von Willebrand's disease: The experience of Hemophilia Region III Treatment Centers over 25 years and review of the literature. Haemoph. Off. J. World Fed. Hemoph. 2007, 13, 685-696. [CrossRef]

29. Girolami, A.; Ruzzon, E.; Tezza, F.; Scandellari, R.; Scapin, M.; Scarparo, P. Congenital FX deficiency combined with other clotting defects or with other abnormalities: A critical evaluation of the literature. Haemoph. Off. J. World Fed. Hemoph. 2008, 14, 323-328. [CrossRef]

30. Zhang, B. Recent developments in the understanding of the combined deficiency of FV and FVIII. Br. J. Haematol. 2009, 145, 15-23. [CrossRef]

31. Watzka, M.; Geisen, C.; Scheer, M.; Wieland, R.; Wiegering, V.; Dörner, T.; Laws, H.J.; Gümrük, F.; Hanalioglu, S.; Ünal, S.; et al. Bleeding and non-bleeding phenotypes in patients with GGCX gene mutations. Thromb. Res. 2014, 134, 856-865. [CrossRef]

32. Ott, R.; Pfeiffer, R.A. Evidence that activities of coagulation factors VII and X are linked to chromosome 13 (q34). Hum. Hered. 1984, 34, 123-126. [CrossRef]

33. Mariani, G.; Bernardi, F. Factor VII Deficiency. Semin. Thromb. Hemost. 2009, 35, 400-406. [CrossRef]

34. Girolami, A.; Ferrari, S.; Cosi, E.; Girolami, B.; Lombardi, A.M. Congenital prothrombin defects: They are not only associated with bleeding but also with thrombosis: A new classification is needed. Hematology 2018, 23, 105-110. [CrossRef]

35. Asselta, R.; Tenchini, M.L.; Duga, S. Inherited defects of coagulation factor V: The hemorrhagic side. J. Thromb. Haemost. 2006, 4, 26-34. [CrossRef]

36. Tizzano, E.F.; Soria, J.M.; Coll, I.; Guzmán, B.; Cornet, M.; Altisent, C.; Martorell, M.; Domenech, M.; del Río, E.; Fontcuberta, J.; et al. The prothrombin 20210A allele influences clinical manifestations of hemophilia A in patients with intron 22 inversion and without inhibitors. Haematologica 2002, 87, 279-285.

37. Kurnik, K.; Escuriola-Ettingshausen, C.; Horneff, S.; Düring, C.; Schobess, R.; Bidlingmaier, C.; Halimeh, S.; Pollmann, H.; Bogdanova, N.; Nowak-Göttl, U. Effects of the factor V G1691A mutation and the factor II G20210A variant on the clinical expression of severe hemophilia A in children-Results of a multicenter studys. Haematologica 2007, 92, 982-985. [CrossRef]

38. Halimeh, S.; Ettingshausen, C.E.; Kurnik, K.; Schobess, R.; Wermes, C.; Pollmann, H.; Kreuz, W.D.; Nowak-Göttl, U. Symptomatic onset of severe hemophilia A in childhood is dependent on the presence of prothrombotic risk factors. Thromb. Haemost. 2001, 85, 218-220. [CrossRef]

39. Marlar, R.A.; Gausman, J.N. Laboratory testing issues for protein C, protein S, and antithrombin. Int. J. Lab. Hematol. 2014, 36, 289-295. [CrossRef]

40. Pavlova, A.; Delev, D.; Pezeshkpoor, B.; Muller, J.; Oldenburg, J. Haemophilia A mutations in patients with non-severe phenotype associated with a discrepancy between one-stage and chromogenic factor VIII activity assays. Thromb. Haemost. 2014, 111, 851-861. [CrossRef] 